\title{
Chemotactic Sensitivity of Escherichia coli to Diffusion Perturbations in Narrow Tubes
}

\author{
Pratap R. Patnaik* \\ Institute of Microbial Technology, Sector 39-A, Chandigarh-160 036, India
}

\begin{abstract}
Diffusion resistance hinders the free chemotactic motility of bacteria through narrow tubes and capillaries. In many such situations, diffusion is significant and often subject to environmental perturbations. This problem has been analyzed through the sensitivities of Escherichia coli cells, a secreted chemoattractant (aspartase) and a nutrient. Differences and similarities among the sensitivity profiles with respect to the three diffusion coefficients reveal useful information about interactions between diffusion and chemotaxis. The results are consistent with the concept of stabilization created by negative feedback, either within the chemosensory network or from outside. Their implications for some real systems are discussed.
\end{abstract}

Keywords: Bacterial chemotaxis, diffusion, narrow tubes, interactions, feedback stabilization.

\section{INTRODUCTION}

Chemotactic movements, i.e. movements guided by chemical stimuli, of bacteria through narrow tubes or capillaries occur in many practical situations. Tissue formation in the peripheral nervous system [1], the controlled delivery of drugs through blood vessels to cure specific diseases [2], the degradation of undesirable chemicals by the preferred movements and metabolization by bacterial colonies [3] and the distributions of populations of cells in capillary flow assays [4] exemplify the variety of applications of chemotaxis through narrow tubes.

Flow through a narrow tube is a critical feature underpinning this analysis; it implies the existence of significant diffusion limitations along the length of the tube but not along its radius. Diffusion resistance creates spatial gradients that modify the paths the cells would have followed if they were free to respond unhindered to chemical stimuli. For completeness, it may be mentioned that chemotactic motions consist of sequences straight line movements, called 'runs', and changes in the directions of the runs, termed 'tumbles'. In the absence of any stimuli, the runs and tumbles are random events, resulting in a uniform distribution of cells in an environment. A chemical stimulus orientates the movements such that a swarm of cells moves either toward a stimulus (for an attractant) or away from it (for a repellant). Diffusion gradients slow down the rate of movement of an ensemble of cells and modify the runs and tumbles of individual cells [5]. These changes are illustrated in Fig. (1), and they differentiate chemotaxis through capillaries or narrow tubes from other situations such as the structural aggregation of cells [6] and product formation in biochemical reactors [7].

While the existence of diffusion resistances and their effects on chemotactic phenomena have been recognized, the effects of perturbations in the diffusivities of either the cells or the chemical stimuli have not been analyzed. Such perturbations are possible in the examples cited at the beginning of

*Address correspondence to this author at the Institute of Microbial Technology, Sector 39-A, Chandigarh-160 036, India;

Fax: +91-172-2690132/585/632; E-mail: pratap@imtech.res.in this section [1-4] because of the ubiquitous presence of both external and internal noise in many multi-cellular processes $[8,9]$. Stochastic perturbations can affect the flow of liquids through tubes and channels and the movements of cells through liquid media, as well as intra-cellular processes that lead to the synthesis of certain proteins. Since the relative movements of cells and of chemoattractants (or chemorepellants) affects the accessibility of these compounds to the cells, and thus regulates the rates of intra-cellular reactions, noise-induced changes in diffusion-affected chemotactic behavior may alter metabolic processes too. Indeed, stochastic resonance between external noise and genetic noise has been invoked to explain the evolution of certain phenotypes that are resistant to interventional treatment $[10,11]$.

In view of the importance of diffusion and its variations on cell motility, the present study analysis the sensitivity of chemotactic behavior to perturbations in the diffusivities of a cell, a chemoattractant and the main carbon substrate in a narrow tube. Owing to the narrowness of the tube, radial variations may be ignored. Therefore, the model presented here has only axial diffusion coupled with chemical reaction. As explained below, Escherichia coli was chosen as the model system.

\section{CHEMOTAXIS MODEL}

Owing to the simplicity of its chemosensory network, its well-understood physiology, its low secretion of endo-toxins, its applications in many useful processes, and the ease of experimental measurements, the chemotaxis of E. coli has been studied by many research workers from different perspectives. Although detailed and complex models have been proposed $[12,13]$, they are difficult to use in real systems, where diffusion and disturbances add to the complexity of both the model and the chemotactic phenomena. A model proposed by Tyson et al. [14] is sufficiently simple and contains most of the relevant features observed in E. coli chemotaxis.

Tyson and coworkers based their model mainly on the experimental observations of Budrene and Berg $[16,17]$ that, in a liquid medium containing intermediates of the TCA cy- 


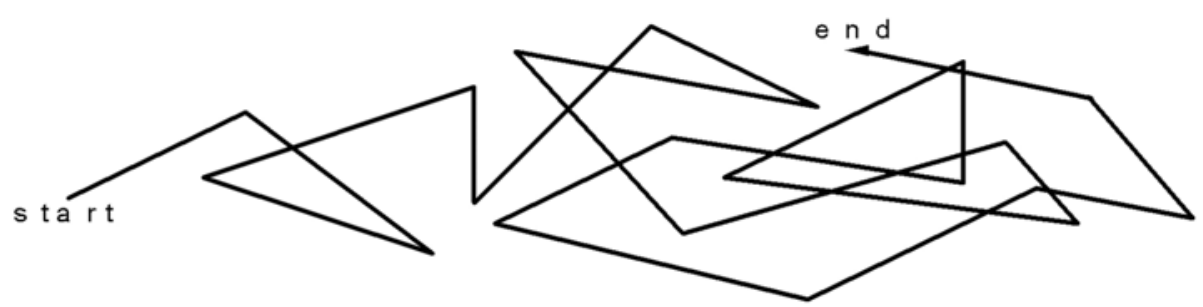

(a) No attractant; random movem ents

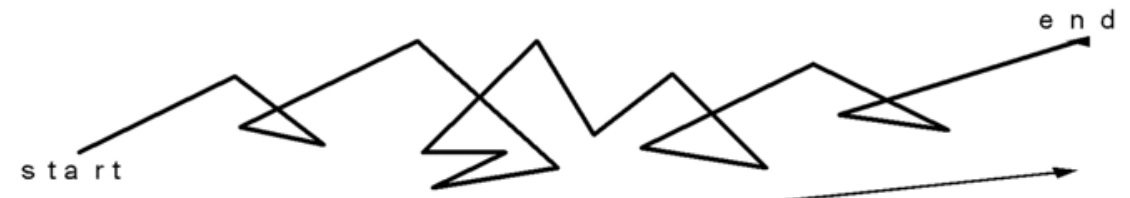

C hem o attractant gradient

D iffus ion gradient

(b) W ith a ttractant; directed movem ents

Fig. (1). Schematic representations of the paths of bacterial cells (a) without a chemoattractant and (b) with a chemoattractant.

cle, E. coli are stimulated to arrange themselves into high density aggregates. The aggregates form different patterns, according to the distribution of the TCA intermediate, of which succinate and fumarate produce the strongest effect. The cells secrete aspartate in response to the stimulant, and Budrene and Berg [16, 17] hypothesized that chemotactic movement toward aspartate was the cause of pattern formation. Interestingly, they also postulated that diffusion counteracts the effect of chemoattraction, a prescient observation whose significance does not seem to have been appreciated until much later. Tyson et al. [14] expressed this background mathematically as:

$$
\frac{\partial \mathrm{b}}{\partial \mathrm{t}}=\mathrm{D}_{\mathrm{b}} \nabla^{2} \mathrm{~b}-\mathrm{k}_{8} \mathrm{n}\left(\frac{\mathrm{k}_{4} \mathrm{~b}^{2}}{\mathrm{k}_{9}+\mathrm{b}^{2}}\right)
$$

Based on the experimental observations of Budrene and Berg [16], Tyson et al. [14] applied two simplifications: (1) the succinate concentration, $\mathrm{b}$, was assumed to be constant and used as a parameter, and (2) $\mathrm{k}_{7}$ and $\mathrm{k}_{8}$ were set to zero. This eliminates Eqn. (4) and reduces Eqns. (2) and (3) to:

$$
\frac{\partial \mathrm{n}}{\partial \mathrm{t}}=\mathrm{D}_{\mathrm{n}} \nabla^{2} \mathrm{n}-\nabla\left[\frac{\mathrm{k}_{1} \mathrm{n}}{\left(\mathrm{k}_{2}+\mathrm{a}\right)^{2}} \nabla \mathrm{a}\right]
$$

From Eqns. (1a)-(1c), they derived the following mathematical representation:

$$
\frac{\partial n}{\partial t}=D_{n} \nabla^{2} n-\nabla\left[\frac{k_{1} n}{\left(k_{2}+a\right)^{2}} \nabla a\right]+k_{3} n\left(\frac{k_{4} b^{2}}{k_{9}+b^{2}}-n\right)
$$

$\frac{\partial \mathrm{a}}{\partial \mathrm{t}}=\mathrm{D}_{\mathrm{a}} \nabla^{2} \mathrm{a}+\mathrm{k}_{5} \mathrm{~b}\left(\frac{\mathrm{n}^{2}}{\mathrm{k}_{6}+\mathrm{n}^{2}}\right)$

$\frac{\partial \mathrm{a}}{\partial \mathrm{t}}=\mathrm{D}_{\mathrm{a}} \nabla^{2} \mathrm{a}+\mathrm{k}_{5} \mathrm{~b}\left(\frac{\mathrm{n}^{2}}{\mathrm{k}_{6}+\mathrm{n}^{2}}\right)-\mathrm{k}_{7} \mathrm{na}$

Subsequently Eqns. (5) and (6) were non-dimensionalized to [15]:

$\frac{\partial \mathrm{z}}{\partial \mathrm{t}}=\mathrm{D}_{\mathrm{z}} \nabla^{2} \mathrm{z}-\alpha \nabla\left[\frac{\mathrm{z}}{(1+\mathrm{c})^{2}}\right] \nabla \mathrm{c}$ 


$$
\frac{\partial \mathrm{c}}{\partial \mathrm{t}}=\mathrm{D}_{\mathrm{c}} \nabla^{2} \mathrm{c}+\mathrm{s}\left(\frac{\mathrm{z}^{2}}{\mu+\mathrm{z}^{2}}\right)
$$

Here $D_{z}=D_{n} / D_{n 0}$ and $D_{c}=D_{a} / D_{0}$ are normalized diffusivities, $c=a / a_{0}$ is the chemoattractant concentration, $s=$ $\mathrm{b} / \mathrm{b}_{0}$ the nutrient (a carbon source) concentration, and $\mathrm{z}=$ $\mathrm{n} / \mathrm{n}_{0}$ that of the bacterial cells. The subscript 0 denotes initial (uniform) values. The values of the parameters are listed in Table 1. Although the values of $\mathrm{k}_{4}, \mathrm{k}_{5}$ and $\mathrm{k}_{9}$ were not known, Tyson et al. [15] did not employ them since the simplified problem consisting of Eqns. (5) and (6) did not contain them.

Lebiedz and Maurer [17] analyzed Tyson et al.'s results $[15,16]$ and noted that nonlinear coupling of three processes through a positive feedback mechanism was the driving force for pattern formation: (a) random migration of the bacteria, (b) diffusion of the chemoattractant and (c) movement of the bacteria in the direction of increasing gradient of the chemoattractant. Then they generalized the observations and proposed the dimensionless model presented below. Note that succinate concentration is no longer assumed to be constant, a more realistic interpretation of many real chemotactic systems [1-4].

$$
\frac{\rho z}{d t}=D_{z} \frac{\partial^{2} z}{\partial x}-\alpha \frac{\rho}{\partial x}\left[\frac{z(1-z / K)}{(1+c)^{2}} \frac{\partial c}{\partial x}\right]+\rho z\left(\frac{\delta s^{2}}{1+s^{2}}-z\right)
$$

$\frac{\partial \mathrm{c}}{\partial \mathrm{t}}=\mathrm{D}_{\mathrm{c}} \frac{\partial^{2} \mathrm{c}}{\partial \mathrm{x}^{2}}+\beta \mathrm{s} \frac{\mathrm{z}^{2}}{\mu+\mathrm{z}^{2}}-\gamma \mathrm{zc}$

$\frac{\partial s}{d t}=D_{s} \frac{\partial^{2} s}{\partial x^{2}}-\kappa z \frac{s^{2}}{1+s^{2}}$

The dimensionless length of the tube spans $\mathrm{x}=0$ to $\mathrm{x}=1$, and at these two ends Eqns. (9)-(12) are subject to the boundary conditions given below [18].

$$
\begin{aligned}
& \frac{\partial z(0, t)}{\partial x}=\frac{\partial z(1, t)}{\partial x}=0 \\
& \frac{\partial c(0, t)}{\partial x}=\frac{\partial c(1, t)}{\partial x}=0 \\
& \frac{\partial s(0, t)}{\partial x}=\frac{\partial s(1, t)}{\partial x}=0
\end{aligned}
$$

The initial conditions are:

$$
\mathrm{t}=0: \mathrm{z}(\mathrm{x}, 0)=\mathrm{s}(\mathrm{x}, 0)=1
$$

and

$\mathrm{t}=0: \mathrm{c}(\mathrm{x}, 0)=0$

While $\mathrm{s}(\mathrm{x}, 0)=\mathrm{z}(\mathrm{x}, 0)=1$ is a result of nondimensionalization, $c(x, 0)$ is set to zero because initially the bacteria have not secreted any chemoattractant. This aspect will be discussed further in a later section.
The first term in Eqns. (9)-(11) describes Fickian diffusion. In Eqn. (9), the second term denotes the chemotactic response and the third is for the growth of the cells. Similarly, the production of chemoattractant (second term in Eqn. (10)) adds to its diffusion transport, while the uptake of chemoattractant by the cells (third term) reduces its extracellular concentration. The rate of change of nutrient concentration (Eqn. (11)) is the difference between the rates of diffusion and consumption. The chemotactic response and the nutrient consumption are both considered to follow saturation kinetics on the basis of previous studies [4, 16, 17]. Lebiedz and Maurer's [18] model reduces to that of Tyson et al. [15] on setting $\gamma=0$ and $\mathrm{K}=\infty$. However, Lebiedz and Maurer set $K=500$, a value sufficiently large to limit the chemotactic flux in regions with high cell densities, thereby ensuring the existence and the uniqueness of a solution to the model [19]. The asymptotic limit of $K=\infty$ unrealistically stops cell motility very early in the growth phase.

\section{SENSITIVITY ANALYSIS}

Many previous studies covering different biological systems such as insulin administration protocols for diabetic patients [20], large biological reactors [21] and bacterial chemotaxis itself [22] have established the value of sensitivity analysis for quantitative information on the robustness of a process to perturbations in certain parameters. The method is described below. To apply it to the present problem, first the parameters have to be identified and then the boundary value problem containing partial differential equations (Eqns.(9)-(14)) has to be converted to an initial value problem comprising ordinary differential equations (ODEs).

Since the subject of this study is the effects of perturbations in the diffusivities of the bacteria, the chemoattractant and the nutrient on the chemotaxis process, the parameters for sensitivity analysis are $D_{z}, D_{c}$ and $D_{s}$. Let $\bar{p}=\left[D_{z} D_{c}\right.$ $\left.D_{s}\right]^{T}$ represent the vector of these parameters, where the superscript $\mathrm{T}$ denotes the transpose. Next, Eqns. (9)-(14) are converted to a set of ODEs by employing orthogonal collocation with Tschebysheff polynomials. This may be done by any of three methods: interior or boundary or mixed collocation. These are described elsewhere [23], with the recommendation that for Neumann boundary conditions of the type shown in Eqns. (12)-(14) mixed collocation should be preferred. Here the two end-points $(x=0$ and $x=1)$ automatically become collocation points and at least one interior point $(0<x<1)$ is chosen. Obviously, greater accuracy is achieved with more collocation points but at the cost of speed and computation effort. Since uniqueness of the solution has already been guaranteed by the choice of K [19] and the relative values of the sensitivities at the inlet the interior and the outlet, at different times, provide sufficient information on the effects of diffusion, one interior point suffices our objective. This point is located at the root of the first order Tschebysheff polynomial, which is $\mathrm{x}=0.577$. With three collocation points, Eqns. (9)-(11) may each be written as a set of three ODEs as described by Villadsen and Stewart [23]. Let $\mathrm{u}_{1}, \mathrm{u}_{2}$ and $\mathrm{u}_{3}$ denote the values of $\mathrm{z}, \mathrm{u}_{4}, \mathrm{u}_{5}$ and $\mathrm{u}_{6}$ those of $\mathrm{c}$, and $\mathrm{u}_{7}, \mathrm{u}_{8}$ and $\mathrm{u}_{9}$ the values of $\mathrm{s}$ at the points $\mathrm{x}=$ $0,0.577$ and 1. Then the ODEs corresponding to Eqns. (9)(11) are as given below. 


$$
\frac{d u_{i}}{d t}=D_{z} \sum_{n=1}^{3} B_{i n} u_{n}+\rho u_{i}\left(\frac{\delta u_{i+6}^{2}}{1+u_{i+6}^{2}}-u_{i}\right)-\quad J_{i k}=\frac{\partial g_{i}}{\partial u_{k}} ; i, k=1(1) 9
$$$$
\Delta(i-2) \alpha\left(\sum_{\mathrm{n}=1}^{3} \mathrm{~A}_{2 \mathrm{n}} \mathrm{u}_{\mathrm{n}+3}\right)\left(\sum_{\mathrm{n}=1}^{3} \mathrm{~A}_{2 \mathrm{n}} \frac{\mathrm{u}_{\mathrm{n}}\left(1-\mathrm{u}_{\mathrm{n}} / \mathrm{K}\right)}{\left(1+\mathrm{u}_{\mathrm{n}+3}\right)^{2}}\right) ; \mathrm{i}=1,2,3
$$$$
\frac{d u_{j}}{d t}=D_{c} \sum_{n=1}^{3} B_{j-3, n} u_{n+3}+\beta u_{j+3}\left(\frac{u_{j-3}^{2}}{\mu+\mu_{j-3}^{2}}\right)-\gamma u_{j-3} u_{j} ; j=4,5,6
$$$$
\frac{\mathrm{du}_{\mathrm{k}}}{\mathrm{dt}}=\mathrm{D}_{\mathrm{s}} \sum_{\mathrm{n}=1}^{3} \mathrm{~B}_{\mathrm{k}-6, \mathrm{n}} \mathrm{u}_{\mathrm{m}+6}-\kappa \mathrm{u}_{\mathrm{k}-6}\left(\frac{\mathrm{u}_{\mathrm{k}}^{2}}{1+\mathrm{u}_{\mathrm{k}}^{2}}\right) \mathrm{k}=7,8,9
$$

In Eqns. (17)-(19), $\Delta(\mathrm{i}-2)$ is the Kronecker delta. Let Eqns. (17)-(19) be written compactly as:

$\frac{\mathrm{d} \overline{\mathrm{u}}}{\mathrm{dt}}=\overline{\mathrm{g}}(\overline{\mathrm{u}}, \overline{\mathrm{p}})$

where

$$
\bar{u}=\left[\begin{array}{lllllllll}
\mathrm{u}_{1} & \mathrm{u}_{2} & \mathrm{u}_{3} & \mathrm{u}_{4} & \mathrm{u}_{5} & \mathrm{u}_{6} & \mathrm{u}_{7} & \mathrm{u}_{8} & \mathrm{u}_{9}
\end{array}\right]^{\mathrm{T}} \text { and }
$$

$\bar{g}=\left[\begin{array}{lllllllll}g_{1} & g_{2} & g_{3} & g_{4} & g_{5} & g_{6} & g_{7} & g_{8} & g_{9}\end{array}\right]^{T}$, with $g_{j}$ denoting the function on the right side of the equation for $\mathrm{du}_{\mathrm{j}} / \mathrm{dt}$. The values of the collocation constants $A_{i j}$ and $B_{i j}(i, j=1,2,3)$ have been tabulated elsewhere [23].

The boundary conditions of Eqns. (12)-(14) may also be expressed in collocated form as:

$$
\begin{aligned}
& \sum_{n=1}^{3} A_{1 n} u_{n}=\sum_{n=1}^{3} A_{3 n} u_{n}=0 \\
& \sum_{n=1}^{3} A_{1 n} u_{n+3}=\sum_{n=1}^{3} A_{3 n} u_{n+3}=0 \\
& \sum_{n=1}^{3} A_{1 n} u_{n+6}=\sum_{n=1}^{3} A_{3 n} u_{n+6}=0
\end{aligned}
$$

The sensitivity of $u_{i}$ with respect any parameter $p_{j}$ is defined as:

$\mathrm{q}_{\mathrm{ij}}=\frac{\partial \mathrm{u}_{\mathrm{i}}}{\partial \mathrm{p}_{\mathrm{j}}} ; \mathrm{i}=1(1) 9 ; \mathrm{j}=1(1) 3$

To compare relative values of the sensitivities, the $\mathrm{q}_{\mathrm{ij}}$ are normalized to:

$$
\sigma_{\mathrm{ij}}=\frac{\mathrm{q}_{\mathrm{ij}} \mathrm{p}_{\mathrm{j}}}{\mathrm{u}_{\mathrm{i}}}
$$

Geevan et al. [20] have shown that the $\sigma_{\mathrm{ij}}$ evolve with time according to:

$$
\frac{\mathrm{d} \sigma_{\mathrm{ij}}}{\mathrm{dt}}=\sum_{\mathrm{k}=1}^{9} \mathrm{~J}_{\mathrm{ik}} \sigma_{\mathrm{kj}}+\frac{\mathrm{p}_{\mathrm{j}}}{\mathrm{u}_{\mathrm{i}}} \frac{\partial \mathrm{g}_{\mathrm{i}}}{\partial \mathrm{p}_{\mathrm{j}}} ; \mathrm{i}=1(1) 9 ; \mathrm{j}=1(1) 3
$$

$\mathrm{J}_{\mathrm{ik}}$ is the (i,k)-th element of the Jacobian matrix of $\overline{\mathrm{g}}$, i.e. the matrix of first partial derivatives:
Since any physical or biological system has a finite positive time constant, it responds to a perturbation after a finite length of time. Therefore, at exactly $\mathrm{t}=0$ all sensitivities are zero, leading to the initial conditions:

$\mathrm{t}=0: \sigma_{\mathrm{kj}}=0 \forall \mathrm{k}, \mathrm{j}$

Since Eqn. (26) contains $u_{i}$ and $g_{j}$, these equations have to be solved together with Eqns. (17)-(19), subject to the initial conditions contained in Eqns. (15), (16) and (28). The unique solution is rendered feasible by being constrained by the transformed boundary conditions in Eqns. (21)-(23). The values of the dimensionless parameters obtained from Table $\mathbf{1}$ are listed in Table $\mathbf{2}$.

Table 1. Values of the Parameters in Eqns. (2)-(4) [14]

\begin{tabular}{|c|c|c|}
\hline Parameter & Value & Units \\
\hline \hline $\mathrm{k}_{1}$ & $3.9 * 10^{-9}$ & $\mathrm{M} \mathrm{cm}^{2} \mathrm{~s}^{-1}$ \\
\hline $\mathrm{k}_{2}$ & $5.0^{*} 10^{*-6}$ & $\mathrm{M}$ \\
\hline $\mathrm{k}_{3}$ & 0 & --- \\
\hline $\mathrm{k}_{4}$ & Unknown & \\
\hline $\mathrm{k}_{5}$ & Unknown & \\
\hline $\mathrm{k}_{6}$ & Unknown & --- \\
\hline $\mathrm{k}_{7}$ & 0 & --- \\
\hline $\mathrm{k}_{8}$ & 0 & \\
\hline $\mathrm{k}_{9}$ & Unknown & $\mathrm{cm}^{2} \mathrm{~s}^{-1}$ \\
\hline $\mathrm{D}_{\mathrm{n}}$ & $2.4 * 10^{-6}$ & $\mathrm{~cm}^{2} \mathrm{~s}^{-1}$ \\
\hline $\mathrm{D}_{\mathrm{a}}$ & $8.9 * 10^{-6}$ & $\mathrm{~cm}^{2} \mathrm{~s}^{-1}$ \\
\hline $\mathrm{D}_{\mathrm{b}}$ & $9.0^{*} 10^{-6}$ & \\
\hline
\end{tabular}

Table 2. Values of the Dimensionless Parameters [18]

\begin{tabular}{|c|c|}
\hline Parameter & Value \\
\hline \hline $\mathrm{D}_{\mathrm{z}}$ & 0.33 \\
\hline $\mathrm{D}_{\mathrm{c}}$ & 1.0 \\
\hline $\mathrm{D}_{\mathrm{s}}$ & 1.0 \\
\hline$\alpha$ & 80.0 \\
\hline$\beta$ & 1.0 \\
\hline$\delta$ & 1.0 \\
\hline$\gamma$ & 0.001 \\
\hline$\kappa$ & 0.001 \\
\hline$\mu$ & 1.0 \\
\hline$\rho$ & 0.001 \\
\hline $\mathrm{K}$ & 500.0 \\
\hline
\end{tabular}



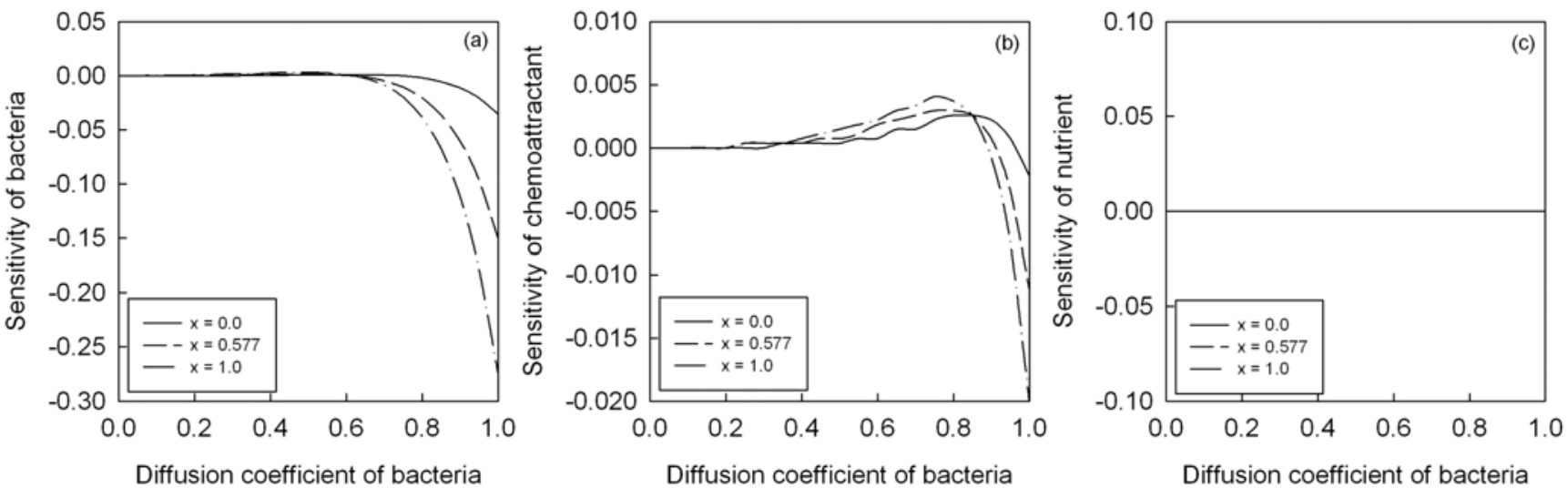

Fig. (2). Sensitivity profiles of $E$. coli, the chemoattractant and the nutrient at different locations in the tube for variations in the normalized diffusion coefficients of the bacteria.

\section{RESULTS AND DISCUSSION}

Sensitivity profiles for the concentrations of E. coli cells, the chemoattractant (aspartate) and the nutrient (a carbon substrate) for the dimensionless parameter values shown in Table $\mathbf{2}$ are plotted in Figs. ( 2 to $\mathbf{4}$ ). The nondimensionalized diffusion coefficients were obtained by normalizing the measured values reported in Table 1 [15]. Considering $\mathrm{D}_{\mathrm{a}}=$ $\mathrm{D}_{\mathrm{n}}$ within experimental accuracy [24], normalization with respect to either leads to the values shown for $D_{z}, D_{c}$ and $D_{s}$ in Table 2.

The nature of the sensitivity plots and the differences among them reveal interesting and useful information about E. coli chemotaxis. We observe first that the bacteria are practically insensitive to fluctuations in all three diffusion coefficients up to nearly six-tenths of the total span of the coefficients. Thereafter the sensitivities decrease continuously and the rate of decrease increases from the inlet to the outlet of the tube. This consistency across all three variables is, however, not maintained for the chemoattractant and the nutrients. While the variation of the sensitivity of the chemoattractant with the diffusion coefficient of $E$. coli is qualitatively similar to that of the bacteria itself, it increases with own its diffusion coefficient and that of the nutrient.

The similarity between the sensitivity profiles of the bacteria and the chemoattractant for variations in the diffusion coefficients of the bacteria is consistent with their synergistic interaction, whereby higher concentrations of the chemoattractant favor faster directed movements of the cells. The contrasting sensitivity profiles of the chemoattractant for variations in the bacterial diffusion coefficient (Fig. 2b), on the one hand, and those of the chemoattractant (Fig. 3b) and the nutrient (Fig. 4b), on the other, may be explained by recognizing that while there is inflow and growth of $E$. coli cells, there are fixed amounts of the two chemicals in the tube. Hence, while the longitudinal transfer of bacterial cells by diffusion may be replenished, rapid diffusion of the nutrient and the chemoattractant increases their availability toward the starting end of the tube, where high concentrations of $E$. coli result in substantial utilization of the chemicals. This in turn reduces the average concentrations of the nutrient and the chemoattractant, thus making them more sensitive to perturbations. The contrasting variations in the sensitivities of $E$. coli cells and those of the other two concentrations is also predicated in the fact that $E$. coli alone displays chemotactic motility and this motility is initiated and regulated by the chemo-attractant.

Two other differences between the sensitivity profiles for the chemoattractant and the bacteria support the explanation provided above. Chemoattractant sensitivities are positive and larger toward the end of the tube than near the inlet, whereas those of $E$. coli are negative and, at a given value of

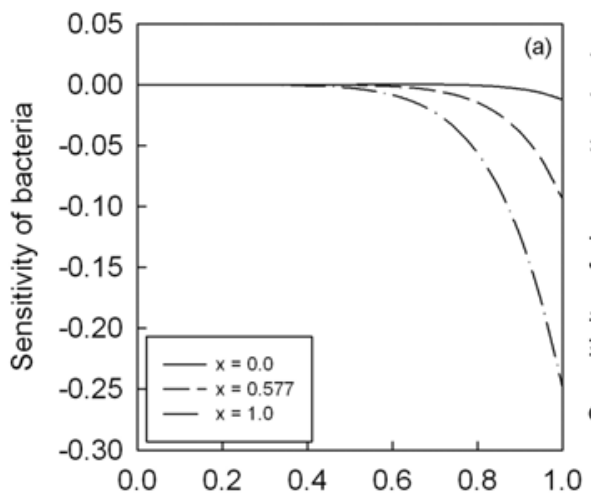

Diffusion coefficient of chemoattractant

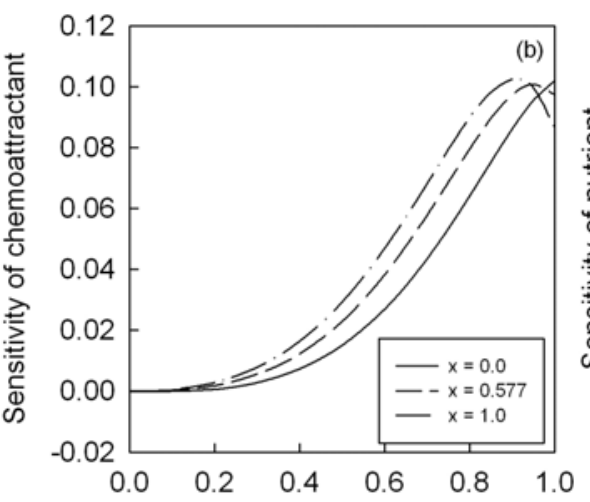

Diffusion coefficient of chemoattractant

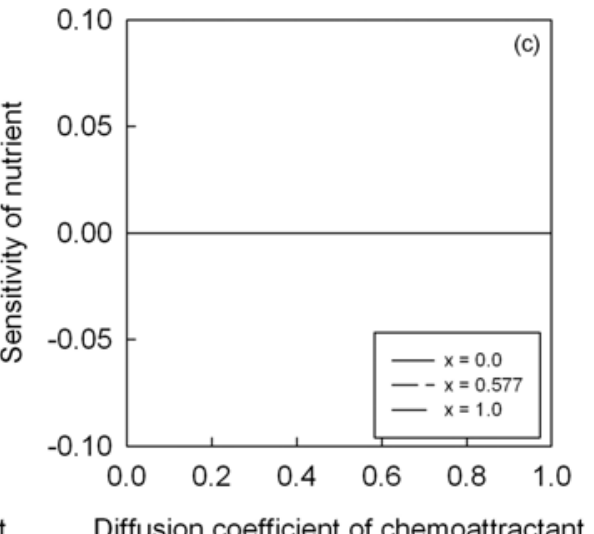

Diffusion coefficient of chemoattractant

Fig. (3). Sensitivity profiles of E. coli, the chemoattractant and the nutrient at different locations in the tube for variations in the normalized diffusion coefficients of the chemoattractant. 

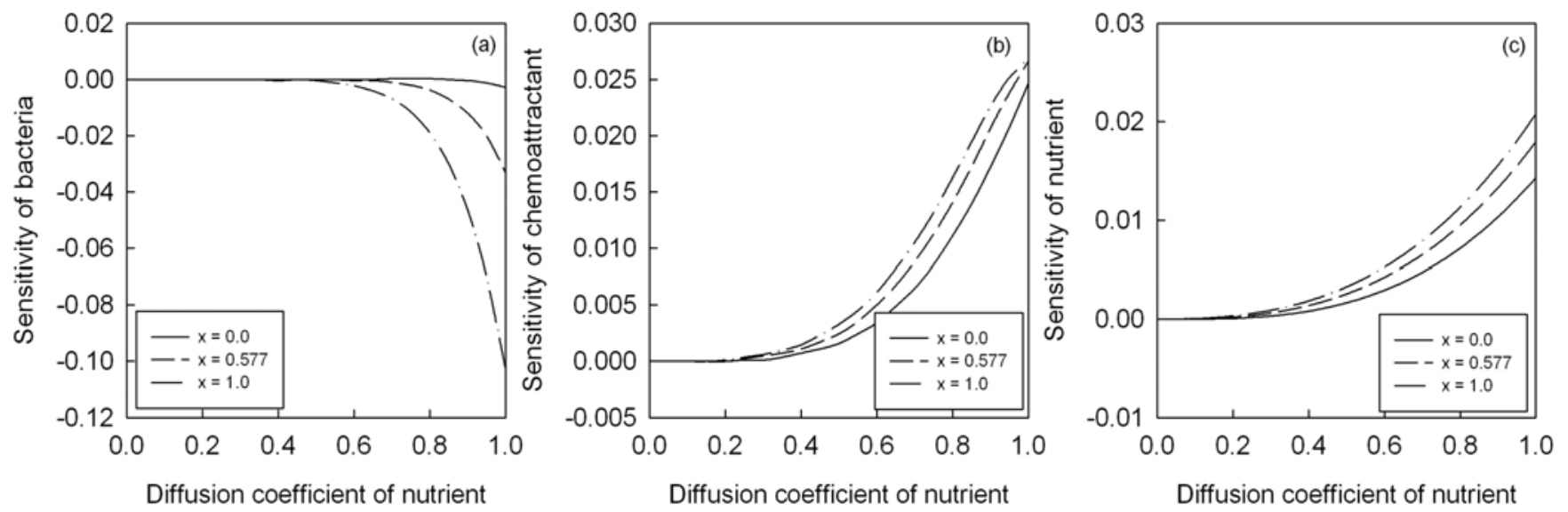

Fig. (4). Sensitivity profiles of E. coli, the chemoattractant and the nutrient at different locations in the tube for variations in the normalized diffusion coefficients of the nutrient.

the diffusion coefficient, they increase (in magnitude) from the inlet to the outlet. These differences are consistent with the fact that bacterial concentrations decrease from the inlet to the outlet whereas those of the chemoattractant have the opposite trend [16-18]. Low sensitivities for large concentrations have also been reported in other microbial systems [21, 25], and have been explained by suggesting that variables present in large concentrations function effectively as sinks that absorb any disturbances, thus rendering them less vulnerable. This explanation is most clearly validated in the present study by the sensitivity plots for the nutrient. This is normally present in much larger amounts than the chemoattractant and the bacteria. Consequently, as might be expected, the nutrient is practically insensitive to perturbations in these two diffusion coefficients whereas its sensitivities with respect to its own diffusion coefficient increase monotonically in the same manner as those of the chemoattractant.

The sensitivity variations observed here are the macroscopic manifestations of more intricate phenomena within the chemosensory network of $E$. coli. This aspect has been discussed recently [26]. The main inference from that analysis that is relevant to the present work is that negative feedback has a stabilizing effect, resulting in greater robustness of a (biological) system to external perturbations. There are two sources of feedback in the chemotaxis of $E$. coli. One is within the chemosensory system and it is provided by the CheB and CheR genes, which control the methylationdemethylation equilibrium of the chemoreceptors in a feedback loop that regulates the concentration of the chemoattractant aspartase $[12,13,22]$. The second source is the diffusion process. Recapitulate that diffusional flow is opposite to that generated by chemoattraction $[15,18]$. Thus, diffusion creates a negative feedback of cells that are moving toward a chemoattractant. Both types of feedback stabilization add to other factors causing the sensitivities of aspartase and the nutrient to be lower than those of the bacteria.

The variations in sensitivity along the length of a tube have important implications. For example, if the bacteria diffuse through pores in the soil toward a chemoattractive pollutant, environmental disturbances at the entrance of a pore may amplify as they propagate toward the end of the pore. Large fluctuations can substantially alter the relative rates of the reactions and the distribution of fluxes in a meta- bolic network. In extreme situations prolonged disturbances may even drive a steady biological system into chaotic behavior [27]. Contrary to this, noise may sometimes promote the creation of order in a microbial system. Chen et al. [9] showed that the extra dynamics introduced by noise may serve an energy source that excites cooperative behavior in a multi-cell system. A therapeutically important area of sensitivity analysis arises from the observation that stochastic resonance between external perturbations and genetic noise has been invoked to explain he evolution of certain phenotypes that are resistant to external interventions such as treatment by antibiotics $[10,28]$. The applications cited here have significant components of both chemotactic and diffusive transport, thus underlining the importance of diffusiondriven sensitivity studies of chemotactic motility.

\section{NOTATION}

All symbols are dimensionless.

c $=$ concentration of chemoattractant

$\mathrm{D}_{\mathrm{c}}=$ diffusion coefficient of chemoattractant

$\mathrm{D}_{\mathrm{s}}=$ diffusion coefficient of nutrient

$\mathrm{D}_{\mathrm{z}}=$ diffusion coefficient of bacteria

$\mathrm{K}=$ maximum permissible local bacterial density

$\mathrm{p}_{\mathrm{j}} \quad \mathrm{j}$-th parameter

$\mathrm{q}_{\mathrm{ij}} \quad=\quad$ sensitivity of $\mathrm{u}_{\mathrm{i}}$ with respect to $\mathrm{p}_{\mathrm{j}}$

$\mathrm{s}=$ concentration of nutrient

$\mathrm{t}=$ time

$\mathrm{u}_{\mathrm{i}}=\mathrm{i}$-th variable

$\mathrm{x}=$ distance along the tube

$\mathrm{z}=$ concentration of bacteria

\section{Greek Letters}

$\alpha=$ rate constant for chemotactic motility

$\beta=$ rate constant for secretion of chemoattractant

$\delta=$ rate constant for uptake of nutrient for cell growth

$\gamma=$ rate constant for uptake of chemoattractant by the cells 
$\kappa=$ rate constant for total consumption of nutrient by the cells

$\mu=$ saturation constant for secretion of chemoattractant

$\rho=$ rate constant for the growth of bacteria

\section{ACKNOWLEDGEMENT}

This is IMTECH communication no.027/2007.

\section{REFERENCES}

[1] N.L. Douarin, and J. Smith, "Development of the peripheral neurons system from the neural crest”, Ann. Rev. Cell Biol., vol. 4, pp. 375-404, 1988.

[2] R.S. Parker, and F.J. Doyle, "Control-relevant modeling in drug delivery", Adv. Drug. Deliv. Rev., vol. 48, pp. 211-228, 2001.

[3] A.M.J. Law, and M.D. Aitken, "Bacterial chemotaxis to naphthalene desorbing from a nonaqeous liquid", Appl. Environ. Microbiol., vol. 69, pp. 5968-5973, 2003.

[4] C. Chiu, and F.C. Hoppensteadt, "Mathematical models and simulations of bacterial growth and chemotaxis in a diffusion gradient chamber", J. Math Biol., vol. 42, pp. 120-144, 2001.

[5] R.L. Bearon, and T.J. Pedley, "Modelling run-and-tumble chemotaxis in a shear flow", Bull. Math Biol., vol. 62, pp. 775-791, 2000.

[6] J. Folkmann, and C. Hauenschild, "Angiogenesis in vitro", Nature, vol. 288, pp. 551-556, 1980.

[7] I.Y. Smets, J.E. Claes, E.J. November, G.P. Bastin, and J.F. Van Impe, "Optimal adaptive control of (bio)chemical reactors: past, present and future", J. Process Control, vol. 14, pp. 795-805, 2004.

[8] P.R. Patnaik, "External, extrinsic and intrinsic noise in cellular systems: analogies and implications for protein synthesis", Biotechnol. Mol. Biol. Rev., vol. 1, pp. 123-129, 2006.

[9] L. Chen, R. Wang, T. Zhou, and K. Aihara, "Noise-induced cooperative behavior in a multicell system", Bioinformatics, vol. 21, pp. 2722-2729, 2005.

[10] M. Kaern, T.C. Elston, W.J. Blake, and J.J. Collins, "Stochasticity in gene expression: from theories to phenotypes", Nat. Rev. Genet., vol. 6 , pp. 451-464, 2005

[11] J.G. Seldman, and C. Seldman, "Transcription factor haploinsufficiency: when half a loaf is not enough", J. Clin. Invest., vol. 109, pp. 451-455, 2002.

[12] C.V. Rao, J.R. Kirby, and A.P. Arkin, "Design and diversity in bacterial chemotaxis: a comparative study in Escherichia coli and Bacillus subtilis”, PloS Biol., vol. 2, pp. 239-252, 2004.
[13] T.-M. Yi, Y. Huang, M.I. Simon, and J. Doyle, "Robust perfect adaptation in bacterial chemotaxis through integral feedback control”, Proc. Natl. Acad. Sci. USA, vol. 97, pp. 4649-4653, 2000.

[14] R. Tyson, S.R. Lubkin, and J.D. Murray, "A minimal mechanism for bacterial pattern formation”, Proc. Roy. Soc. Lond. B, vol. 266, pp. 299-304, 1999.

[15] R. Tyson, S.R. Lubkin, and J.D. Murray, "Model and analysis of chemotactic bacterial patterns in a liquid medium", J. Math Biol., vol. 38, pp. 359-375, 1999.

[16] E.O. Budrene, and H.C. Berg, "Complex patterns formed by motile cells of Escherichia coli”, Nature, vol. 349, pp. 630-633, 1991.

[17] E.O. Budrene, and H.C. Berg, "Dynamics of formation of symmetrical patterns by chemotactic bacteria", Nature, vol. 376, pp. 49-53, 1995.

[18] D. Lebiedz, and H. Maurer, "External optimal control of selforganisation dynamics in a chemotaxis reaction diffusion system", IEE Systems Biol., vol. 2, pp. 222-229, 2004.

[19] T. Hillen, and K. Painter, "Global existence for a parabolic chemotaxis model with prevention of overcrowding", Adv. Appl. Math., vol. 26, pp. 280-301, 2001.

[20] C.P. Geevan, J. Subba Rao, G. Subba Rao, and J.S. Bajaj, "A mathematical model for insulin kinetics III: sensitivity analysis of the model", J. Theor. Biol., vol. 147, pp. 255-263, 1990.

[21] P.R. Patnaik, "Sensitivity propagations in response to start-up perturbations during batch fermentations with complementary cultures”, Chem. Eng. Res. Des., vol. 85(A7), pp. 1079-1085, 2007.

[22] H. Kitano, "Biological robustness", Nat. Rev. Genet., vol. 5, pp. 826-837, 2004

[23] J.V. Villadsen, and W.E. Stewart, "Solution of boundary-value problems by orthogonal collocation", Chem. Eng. Sci., vol. 22, pp. 1483-1501, 1967.

[24] H.C. Berg, Random walks in biology. Princeton, NJ: Princeton Univ. Press, 1983.

[25] P.R. Patnaik, "Steady state performance and sensitivity of a continuous recombinant fermentation with plasmid copy number multiplicity", Ind. J. Biotechnol., vol. 1, pp. 263-269, 2002.

[26] P.R. Patnaik, "Robustness analysis of the E. coli chemosensory system to perturbations in chemoattract concentrations", Bioinformatics, vol. 23, pp. 875-881, 2007.

[27] P.R. Patnaik, "Hybrid filtering to rescue stable oscillations from noise-induced chaos in continuous cultures of budding yeast", FEMS Yeast Res., vol. 6, pp. 129-138, 2006.

[28] N.Q. Balaban, J. Merrin, R. Chait, L. Kowalik, and S. Leibler S, "Bacterial persistence as a phenotypic switch", Science, vol. 305, pp. 1622-1625, 2004. 